POS $\quad \begin{aligned} & \text { PROCEEDINGS } \\ & \text { OF SCIENCE }\end{aligned}$

\title{
NNLO predictions for jets and V+jet at the LHC
}

\section{James Currie}

Institute for Particle Physics Phenomenology, Durham University, Durham, DH1 3LE, UK

E-mail: james.currie@durham.ac.uk

\section{Aude Gehrmann-De Ridder}

Institute for Theoretical Physics, ETH, CH-8093 Zürich, Switzerland

Physik-Institut, Universität Zürich, Winterthurerstrasse 190, CH-8057 Zürich, Switzerland

E-mail: gehraephys.ethz.ch

\section{Thomas Gehrmann}

Physik-Institut, Universität Zürich, Winterthurerstrasse 190, CH-8057 Zürich, Switzerland

E-mail: thomas.gehrmanneuzh.ch

\section{Nigel Glover}

Institute for Particle Physics Phenomenology, Durham University, Durham, DH1 3LE, UK

E-mail: e.w.n.glover@durham.ac.uk

\section{Alexander Huss*}

Theoretical Physics Department, CERN, 1211 Geneva 23, Switzerland

E-mail: alexander.huss@cern.ch

\section{Joao Pires}

Centro de Física Teórica de Partículas - CFTP, Instituto Superior Técnico IST, Universidade de Lisboa, Av. Rovisco Pais, P-1049-001 Lisboa, Portugal

E-mail: joao.ramalho.piresetecnico.ulisboa.pt

\section{Duncan Walker}

Institute for Particle Physics Phenomenology, Durham University, Durham, DH1 3LE, UK

E-mail: duncan.m.walker@durham.ac.uk

The production of jets and electroweak gauge bosons in association with a jet, $V+$ jet, constitute two important classes of standard-candle processes at the LHC. Owing to the large event rate and the direct sensitivity to the strong coupling constant and the gluon PDF, they provide an ideal testing ground for our understanding of perturbative QCD in a hadron-collider environment. NNLO QCD corrections are now available for these processes and allow for a detailed study of these corrections in phenomenological applications and their impact on the interpretation of experimental data.

XXVI International Workshop on Deep-Inelastic Scattering and Related Subjects (DIS2018) 16-20 April 2018

Kobe, Japan

\footnotetext{
*Speaker.
} 


\section{V+jet Production and Related Observables}

The production of electroweak gauge bosons $\left(V=\gamma, \mathrm{Z}, \mathrm{W}^{ \pm}\right)$are among the most important "standard candles" at the LHC due to their large production cross section as well as their clean experimental signature. By requiring and additional jet in the final state, one largely retains these properties while gaining a direct sensitivity to the strong coupling constant $\alpha_{\mathrm{s}}$ and the gluon PDF. As such, $V+$ jet production represents a clean testing ground for perturbative QCD predictions with a wide range of applications.

An observable that is closely related to the $V+$ jet process is the inclusive $p_{\mathrm{T}}^{V}$ spectrum of the gauge bosons. This is due to the fact that a non-vanishing value for $p_{\mathrm{T}}$ implicitly requires some hadronic recoil to balance the transverse momentum. As a result, the NNLO QCD corrections that are now available for all $V+$ jet processes [1-5] can be used to predict the $p_{\mathrm{T}}^{V}$ spectra of the gauge bosons to genuine NNLO $\mathscr{O}\left(\alpha_{\mathrm{s}}^{3}\right)$ accuracy.
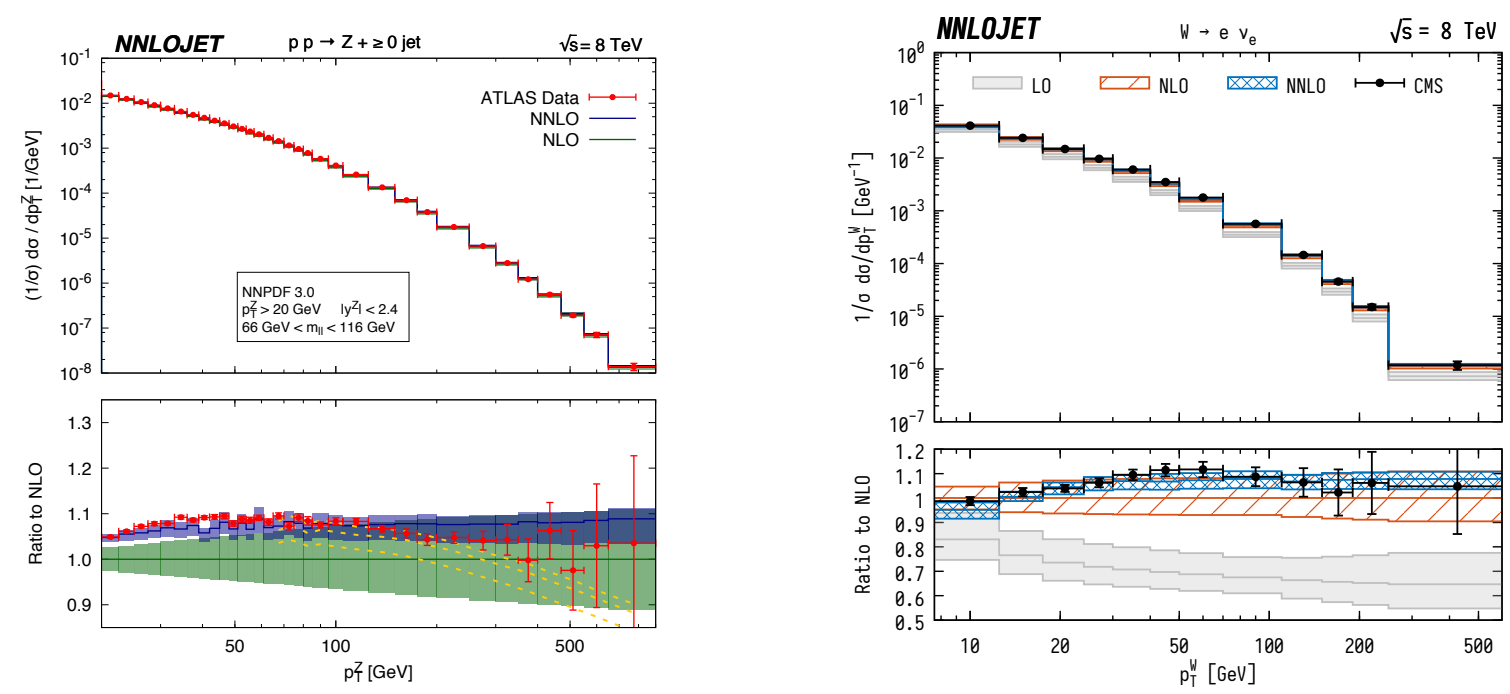

Figure 1: The Z-boson (left) and W-boson (right) transverse momentum distribution up to NNLO in QCD as computed in Refs. [6,7] and [8], respectively.

Figure 1 shows the fixed-order QCD predictions up to NNLO for the $p_{\mathrm{T}}^{V}$ spectra in Z-boson (left) and W-boson (right) production. In both cases, we observe sizeable NNLO corrections of up to $10 \%$ that further result in a visible distortion of the shape. A comparison with the measurements by ATLAS and CMS shows an improved description of the data after including the NNLO corrections, which also exhibit a reduced scale uncertainty by typically a factor of two compared to the respective predictions at NLO. We note that in the limit of small transverse momenta, $p_{\mathrm{T}} \rightarrow 0$, fixed-order predictions become unreliable due to the divergent behaviour of logarithms $\sim \alpha_{\mathrm{s}}^{n} \log ^{k}\left(p_{\mathrm{T}} / M\right)$ that appear in the calculation. In this regime, an all-order resummation of these large logarithmic terms becomes mandatory in order to obtain reliable predictions down to small $p_{\mathrm{T}}$. Very recently, a matching between $\mathrm{N}^{3} \mathrm{LL}$ resummation and the fixed-order NNLO calculation discussed above has been achieved both for H-boson and Z-boson production [9].

As can be seen from Fig. 1, the QCD corrections are very similar between the Z-boson and Wboson production processes. This behaviour is not surprising since higher-order strong interactions 

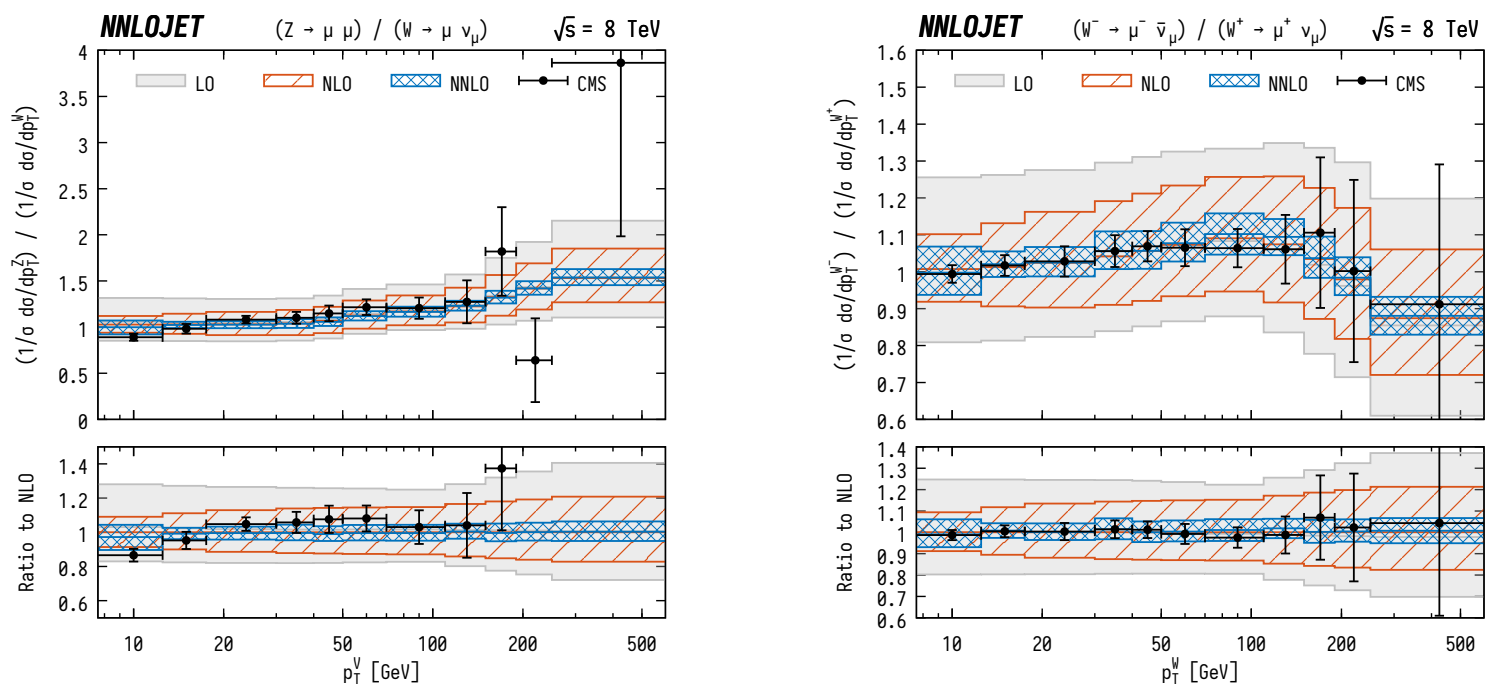

Figure 2: Ratios of transverse momentum spectra for $\mathrm{Z} / \mathrm{W}^{ \pm}$(left) and $\mathrm{W}^{-} / \mathrm{W}^{+}$(right) as computed in Ref. [8].

do not interfere directly with the production of colour-neutral states. As such, one can expect the ratio between different $p_{\mathrm{T}}^{V}$ spectra to be quite stable with respect to QCD corrections. Such ratios are important quantities not only for constraining PDFs but they also enter indirectly in important precision measurements such as the extraction of the W-boson mass. Figure 2 shows predictions at different perturbative orders for the $\mathrm{Z} / \mathrm{W}^{ \pm}$(left) and $\mathrm{W}^{-} / \mathrm{W}^{+}$(right) ratios. The correction factors are found to be remarkably stable at around one, further supporting the similarity between the $V$-production processes. The inclusion of higher-order corrections mainly manifests itself in the reduction of theory uncertainties, which are estimates via an uncorrelated variation of the renormalisation and factorisation scales between the numerator and the denominator. Going from NLO to NNLO, we observe a reduction by typically more than a factor of two across the entire $p_{\mathrm{T}}$ spectrum under consideration with a residual scale uncertainty at the level of 5-10\%.

Another important quantity in gauge-boson production are the angular coefficients, which allow for a detailed study of the QCD production dynamics and the polarisation of the intermediate gauge boson. This is achieved by a decomposition of the lepton angular dependence in terms of spherical harmonics, which encapsulate the lepton kinematics, such that the coefficients $A_{i}$ of this decomposition carry the information of the production dynamics. A particularly interesting relation in this respect is the so-called Lam-Tung relation, which predicts $\left(A_{0}-A_{2}\right)=0$. This relation is only violated starting from $\mathscr{O}\left(\alpha_{\mathrm{s}}^{2}\right)$ in the Drell-Yan process and thus makes it particularly interesting to study QCD predictions. Figure. 3 shows a comparison of different perturbative orders in QCD for the observables $A_{0}, A_{2}$, and $\left(A_{0}-A_{2}\right)$ together with the measured data by ATLAS. We can clearly observe a strong tension between data and theory for the difference $\left(A_{0}-A_{2}\right)$ at NLO, while the NNLO corrections give rise to sizeable corrections that lie outside of the NLO scale uncertainty bands. Inspecting the coefficients $A_{0}$ and $A_{2}$ separately, the effect is mainly driven by the coefficient $A_{2}$ which receives large perturbative corrections while $A_{0}$ remains very stable. The tension with the data is largely resolved by the inclusion of NNLO corrections, with a $\chi^{2} / N_{\text {dat }} \sim 4.89$ at NLO that reduces to $\chi^{2} / N_{\text {dat }} \sim 1.75$ at NNLO. 

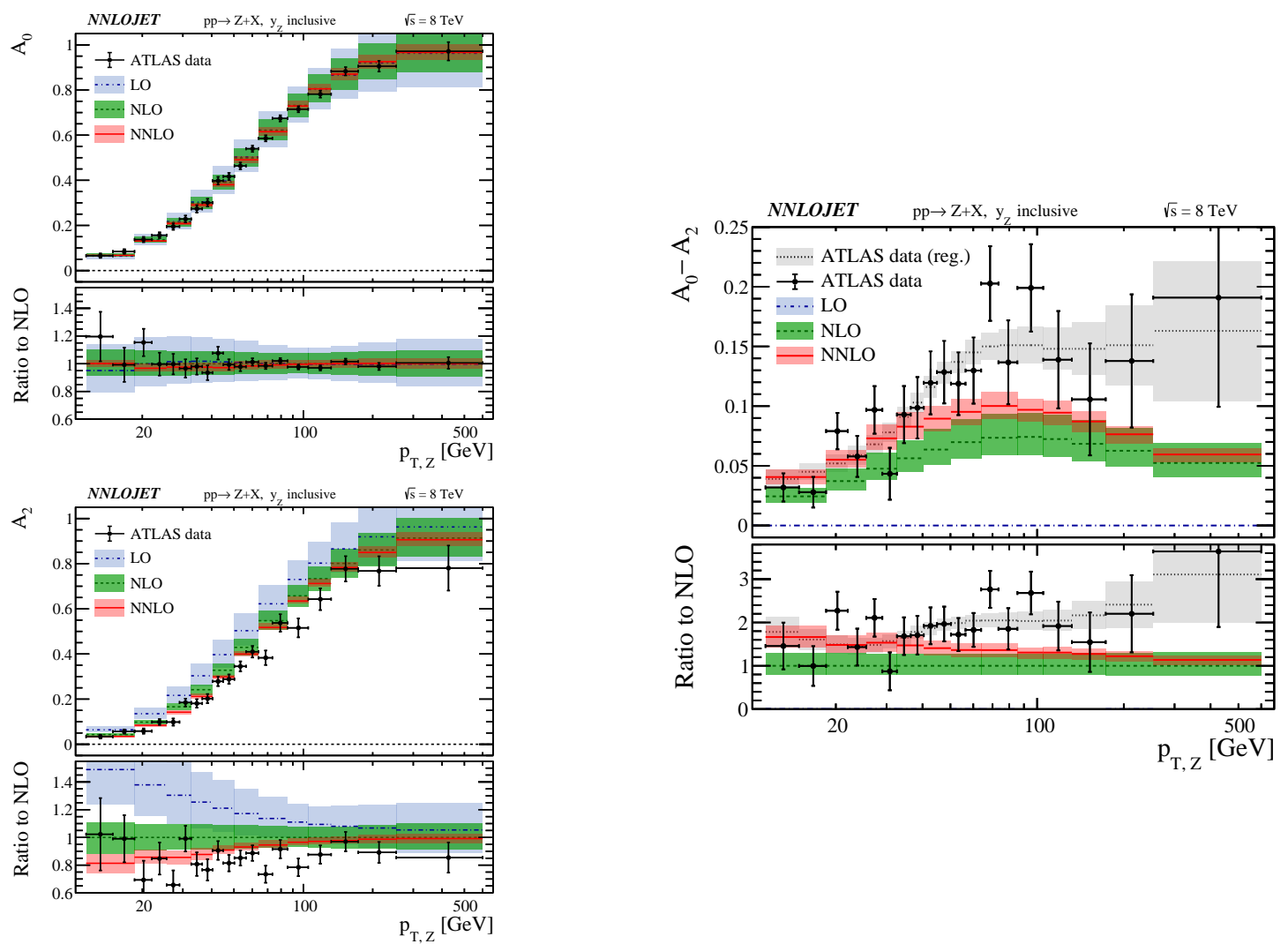

Figure 3: Higher-order corrections to the angular coefficients $A_{0}$ and $A_{2}$ (left) and their difference (right). Figures taken from Ref. [10].

\section{Jet Production at the LHC}

Given the nature of a hadron-hadron collider environment, jets are produced in abundance at the LHC and allow for a detailed study of QCD predictions across a wide range of kinematics. Not only does this process deliver important constraints in PDF fits but it also allows to study the running of $\alpha_{\mathrm{s}}$ and further represents an important background in searches for physics beyond the Standard Model. The recent completion of the NNLO QCD corrections [11, 12] will allow for many important phenomenological applications as well as detailed theory studies based on the three perturbative orders that are now available.

One of the most basic observables in jet production is the inclusive $p_{\mathrm{T}}$ spectrum, where every reconstructed jet in an event contributes individually to the observable. As a result, it is possible to choose among many different scales with event-based scales defining a common scale for all jets in the event and jet-based scales setting a scale for each jet individually. The two scales $\mu=p_{\mathrm{T}, 1}$ (transverse momentum of the leading jet) and $\mu=p_{\mathrm{T}}$ (transverse momentum of the individual jets) are the two most common choices, where the former constitutes an event-based and the latter a jet-based scale. A comparison of the two scales is shown in Fig. 4 at NLO (left) and NNLO (right). We observe that in the high- $p_{\mathrm{T}}$ tail, the two scale choices coincide as one is more and more dominated by back-to-back dijet configurations where $p_{\mathrm{T}, 1}=p_{\mathrm{T}}$. In this region, the NNLO corrections lead to a reduction of scale uncertainties and further exhibit a good perturbative convergence with 

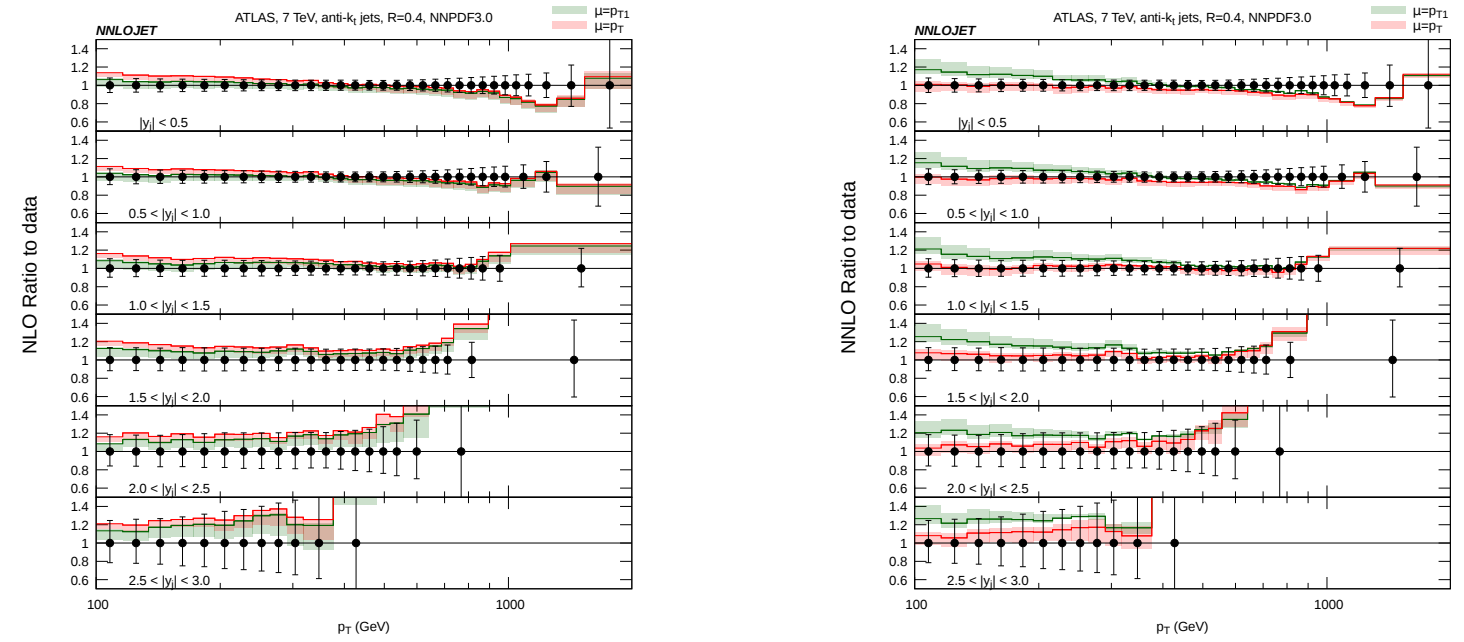

Figure 4: Comparison of the scale choices $\mu=p_{\mathrm{T}, 1}$ and $\mu=p_{\mathrm{T}}$ in inclusive jet production at NLO (left) and NNLO (right).

the NNLO prediction lying within the uncertainty estimate of the previous order. In the low- $p_{\mathrm{T}}$ region, however, larger differences are observed between the two scale choices where the impact of changing the scale gives rise to differences beyond the respective uncertainty estimates. This behaviour is even more pronounced at NNLO than at NLO and is an indication of a potential infrared sensitivity of the inclusive jet observable itself. In order to understand the source of this problem, a more detailed study including different scales and cone sizes will be necessary.

The dijet production process is not plagued by such instability issues as can be seen for the dijet invariant mass distribution $\left(m_{j j}\right)$ in the left-hand plots of Fig. 5. Both in the central (top) and forward (bottom) $y^{*} \equiv\left(y_{j_{1}}-y_{j_{2}}\right) / 2$ regions, we observe a convergent behaviour of the perturbative series for the two scales $\mu=m_{j j}$ and $\mu=\left\langle p_{\mathrm{T}}\right\rangle$. Although the scale $\mu=\left\langle p_{\mathrm{T}}\right\rangle$ exhibits much larger NLO corrections together with bigger scale uncertainty bands, at NNLO the different scale choices show a tendency to converge towards the same answer. In the right plot of Fig. 5 we show the differential distribution in $m_{j j}$ for the scale choice $\mu=m_{j j}$ at NLO and NNLO. A comparison with the measurement shows an improved description at NNLO, in particular in the central $y^{*}$ region, where the corrections induce a change in the shape of the distribution. Overall, the scale uncertainties are reduced substantially in the forward $y^{*}$ and high- $m_{j j}$ regions.

\section{References}

[1] R. Boughezal, C. Focke, X. Liu, and F. Petriello Phys. Rev. Lett. 115 (2015), no. 6 062002, [arXiv:1504.02131].

[2] A. Gehrmann-De Ridder, T. Gehrmann, E. W. N. Glover, A. Huss, and T. A. Morgan Phys. Rev. Lett. 117 (2016), no. 2 022001, [arXiv: 1507.02850 ].

[3] R. Boughezal, J. M. Campbell, R. K. Ellis, C. Focke, W. T. Giele, X. Liu, and F. Petriello Phys. Rev. Lett. 116 (2016), no. 15 152001, [arXiv:1512.01291]. 

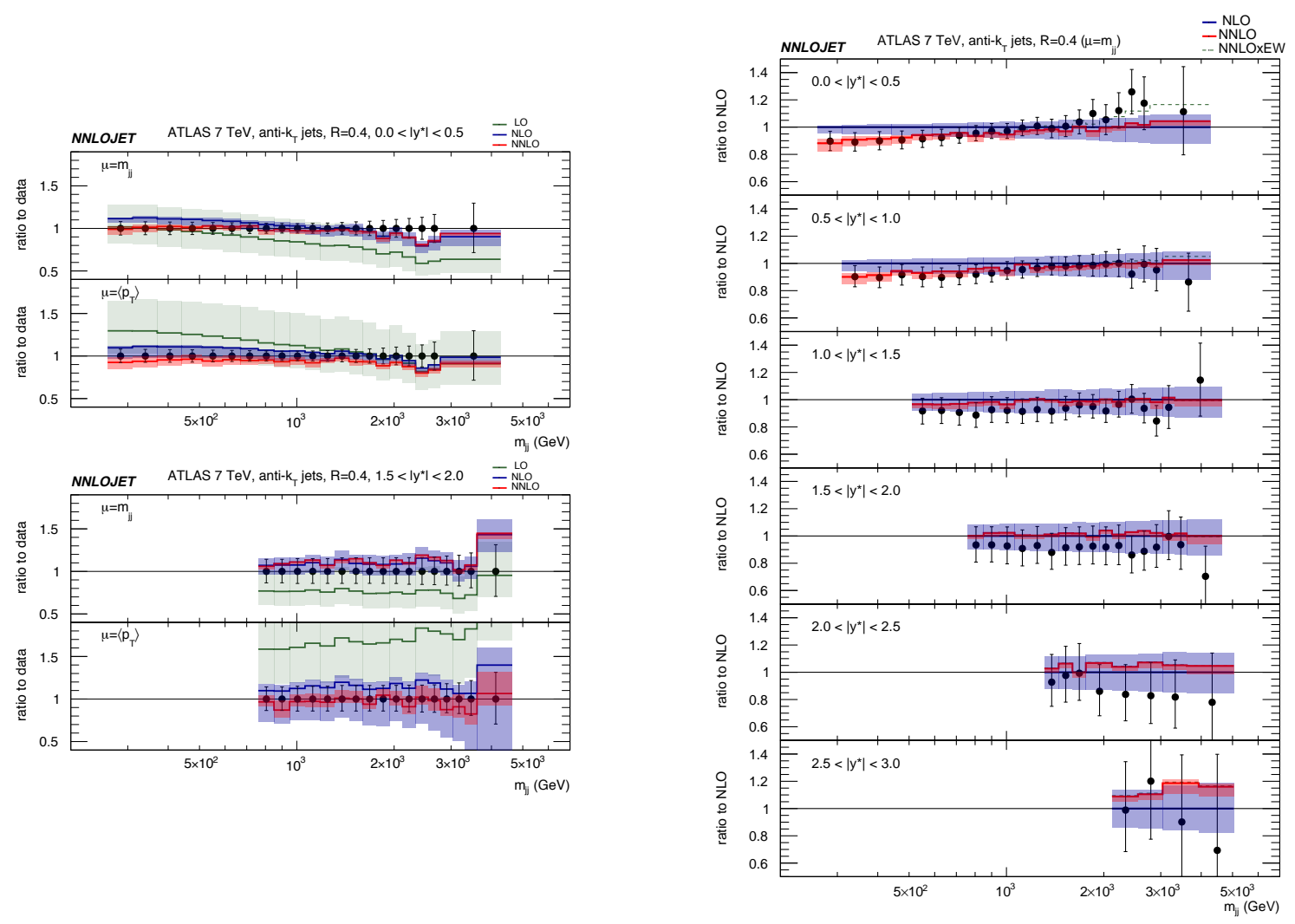

Figure 5: A comparison of the two scale choices $\mu=m_{j j}$ and $\mu=\left\langle p_{\mathrm{T}}\right\rangle$ in dijet production (left). Predictions for the double-differential distribution in $m_{j j}$ and $y^{*}$ in dijet production using the scale $\mu=m_{j j}$ as computed in Ref. [12].

[4] J. M. Campbell, R. K. Ellis, and C. Williams Phys. Rev. Lett. 118 (2017), no. 22 222001, [arXiv:1612.04333].

[5] J. M. Campbell, R. K. Ellis, and C. Williams Phys. Rev. D96 (2017), no. 1014037 , [arXiv:1703.10109].

[6] A. Gehrmann-De Ridder, T. Gehrmann, E. W. N. Glover, A. Huss, and T. A. Morgan JHEP 07 (2016) 133, [arXiv:1605.04295].

[7] A. Gehrmann-De Ridder, T. Gehrmann, E. W. N. Glover, A. Huss, and T. A. Morgan JHEP 11 (2016) 094, [arXiv:1610.01843].

[8] A. Gehrmann-De Ridder, T. Gehrmann, E. W. N. Glover, A. Huss, and D. M. Walker Phys. Rev. Lett. 120 (2018), no. 12 122001, [arXiv: 1712.07543 ].

[9] P. F. Monni these proceedings.

[10] R. Gauld, A. Gehrmann-De Ridder, T. Gehrmann, E. W. N. Glover, and A. Huss JHEP 11 (2017) 003, [arXiv:1708.00008].

[11] J. Currie, E. W. N. Glover, and J. Pires Phys. Rev. Lett. 118 (2017), no. 7072002 , [arXiv:1611.01460].

[12] J. Currie, A. Gehrmann-De Ridder, T. Gehrmann, E. W. N. Glover, A. Huss, and J. Pires Phys. Rev. Lett. 119 (2017), no. 15 152001, [arXiv:1705.10271]. 